\title{
Reevaluation of the conclusion that IRES-activity reported within the 5 ' leader of the TIF4631 gene is due to promoter activity
}

\author{
VINCENT P. MAURO, GERALD M. EDELMAN, and WEI ZHOU \\ Department of Neurobiology, The Scripps Research Institute, and The Skaggs Institute for Chemical Biology, La Jolla, California 92037, USA
}

\begin{abstract}
We previously reported that the $5^{\prime}$ leader of the mRNA-encoding initiation factor elF4G in Saccharomyces cerevisiae can function as a translational enhancer and as an internal ribosome entry site (IRES) when tested in cells. However, Verge and colleagues recently suggested that this sequence does not facilitate translation initiation, but inhibits translation in vitro and has promoter activity when tested in cells. We disagree with these conclusions and respond by showing that the data are most consistent with an internal initiation mechanism.
\end{abstract}

Keywords: IRES; TIF4631; eIF4G; translation; mRNA

Verge et al. (2004), in the February issue of RNA, reported that contrary to our earlier publication (Zhou et al. 2001), the $5^{\prime}$ leader of the TIF4631 mRNA does not enhance translation initiation or contain an IRES. The authors assert that they have identified a promoter, located 36-112 nucleotides upstream of the translation initiation site, and concluded in their abstract, "The data show that the IRES activity reported earlier is due to this promoter". However, in our study, the IRES activity was localized primarily to a segment of the $5^{\prime}$ leader that is completely independent of this putative promoter (nucleotides 250-390, see Fig. 1). Moreover, we showed that a segment of the TIF4631 gene containing the putative promoter had a background level of activity when tested in the intercistronic region of a dicistronic mRNA (construct p150[375-508]). This low level of activity was $\sim 1 \%$ of the activity of the full-length $5^{\prime}$ leader.

The notion that the IRES activities observed in our study were derived from the translation of a shorter monocistronic mRNA is also not consistent with the high levels of activity reported in our paper. By accounting for the differences in luminescence between the Renilla and Photinus luciferases, we estimate that the second cistron expressed $\sim 40 \%$ as much protein as the first cistron in constructs containing the TIF4631 5' leader. Inasmuch as the dicis-

Reprint request to: Vincent P. Mauro, The Scripps Research Institute, 10550 North Torrey Pines Road, La Jolla, CA 92037, USA; e-mail: vmauro@scripps.edu; fax: (858) 784-2646.

Article and publication are at http://www.rnajournal.org/cgi/doi/ 10.1261/rna.7160404. tronic mRNA was easily detected in our study, a shorter monocistronic transcript should also have been easily identified; however, we found no evidence for such a transcript, even when our Northern blots were overexposed.

Verge et al. (2004) reported promoter activity within the 5 ' leader of the TIF4631 mRNA; however, they did not directly test for this activity, for example, by testing the fragment in question in a promoterless vector or with Northern blots. Instead, indirect methods were used to assess promoter activity. For example, mRNA species were detected by PCR analysis. This is a highly sensitive technique that will detect trace amounts of RNA; however, their experiments were not performed in a quantitative manner, consequently it is not possible to compare mRNA levels or to draw conclusions about the production of a monocistronic mRNA from this data. This limitation is indicated by an internal inconsistency in their data: In transformed cells for which the expression of the dicistronic mRNAs was not induced, the authors obtained a PCR band corresponding to the first cistron (Renilla luciferase) of the control dicistronic mRNA (see their Fig. 3, lane 9). They did not, however, obtain a PCR product for this cistron with a construct containing the eIF4G 5' leader (Fig. 3, lane 1). Yet, Renilla luciferase activities were approximately twice as high in cells containing the eIF4G construct than in cells containing the control construct (see their Fig. 2, CW04 cells, constructs 1 and 2, minus induction). These results indicate that the PCR bands in Figure 3 do not reflect mRNA concentrations in these cells. 


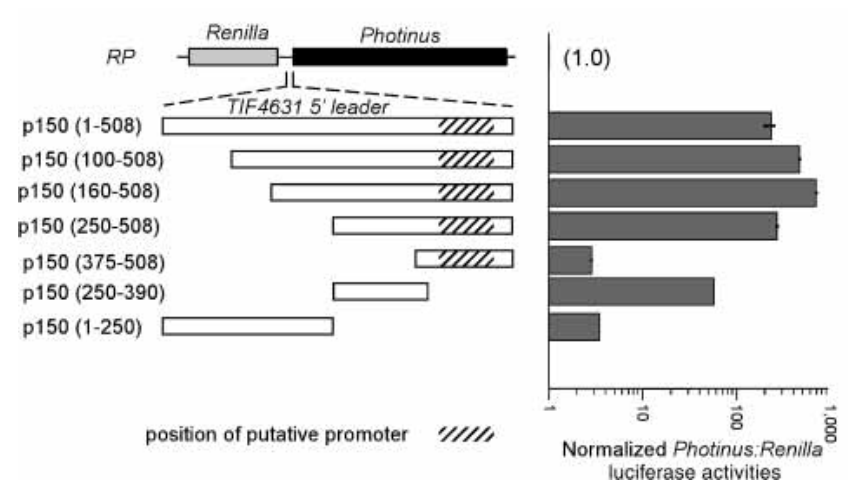

FIGURE 1. Deletion and fragment analysis of the TIF4631 IRES in Saccharomyces cerevisiae cells shows that IRES activity does not colocalize with the putative promoter activity identified by Verge et al. (2004). (This figure is adapted from Figure 5 in Zhou et al. 2001, (C) 2001 National Academy of Sciences, USA.) Schematic representations of the dicistronic constructs used in this analysis are indicated. Constructs are based on the $R P$ vector, which contains Renilla luciferase as the first cistron and Photinus luciferase as the second cistron. The transcription of the dicistronic mRNAs is under the control of the GAL1 promoter. Inserts include the full-length TIF4631 5' leader sequence $[\mathrm{p} 150(1-508)]$ in addition to $5^{\prime}$ deletions and fragments of this sequence. The construct designations indicate, in parentheses, the exact nucleotide sequences present in each construct. The location of the putative promoter identified by Verge et al. (2004) is indicated as a hatched bar. Yeast strain EGY48 (CLONTECH) was transformed with the constructs indicated and grown in glucose-containing medium. After $24 \mathrm{~h}$, cells were washed with $\mathrm{H}_{2} \mathrm{O}$ and cultured in medium containing $2 \%$ galactose and $1 \%$ raffinose. Luciferase activities were determined $3 \mathrm{~h}$ after galactose induction of dicistronic mRNA transcription. IRES activities are represented on a logarithmic scale as ratios of Photinus luciferase activity to Renilla luciferase activity. Values represent activities that have been normalized to those of the $R P$ vector. Horizontal bars, SEM.

The other indirect method used to assess promoter activity comes from Figure 4, in which the authors suggest that the putative promoter linked to initiation factor eIF4G can rescue a strain lacking chromosomal copies of TIF4631 and TIF4632. However, it is possible that the constructs were altered by mutation, rearrangement, or recombination events. In addition, the level of promoter activity required to rescue these cells was not determined. Even if these cells were rescued by what appears to be a weak promoter, this result is not informative regarding the activity or location of the native promoter.

The authors identified a short primer extension product, which they determined to be the transcription start site, but provided no additional evidence to determine whether the bands were authentic transcription start sites or were due to stalling. They also identified two longer primer extension products $(-390$ and -580$)$, one of which corresponds to that previously reported by Goyer et al. (1993), and that was used in our study. In their discussion, Verge et al. (2004) concede that "we cannot exclude that additional longer forms of the TIF4631 mRNA are produced and perhaps translated by internal initiation". However, in their abstract, they contradicted this by strongly ruling out these possibilities.
The fact that the translation of the second cistron did not increase when the transcription of the dicistronic mRNA was induced (Verge et al. 2004, Fig. 2) appears at first to be definitive evidence that the TIF4631 5' leader does not function as an IRES. We have also performed this analysis, and on the contrary, observed an increase in second cistron expression that is proportional to the increased expression of the first cistron (Fig. 2). This data was obtained using a dicistronic construct with a stable stem-loop structure $5^{\prime}$ of the first cistron to minimize any potential contribution of this cistron; however, comparable results were obtained with constructs lacking the stem-loop structure.

We believe that the discrepancy between our results and those presented by Verge et al. (2004) may be due to technical differences between the experiments. For example, in Figure 2 of their paper, Verge et al. (2004) reported Renilla
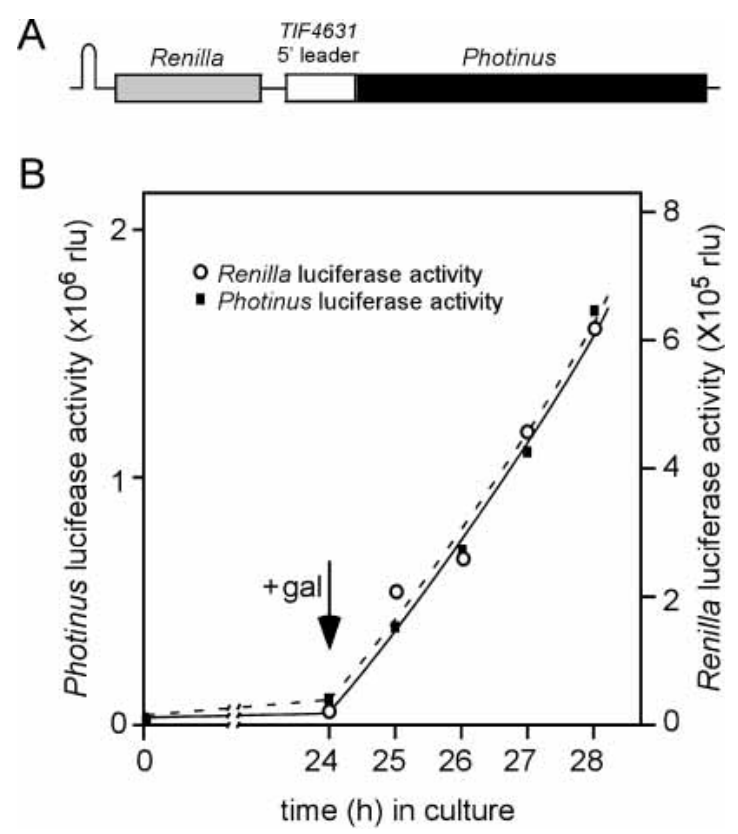

FIGURE 2. Analysis of S. cerevisiae TIF4631 5' leader in the intercistronic region of a dicistronic reporter mRNA following galactose induction of the dicistronic mRNA. (A) Schematic representation of the dual luciferase dicistronic mRNA. This construct $[\mathrm{p} 150(1-508) / R P \mathrm{~h}]$ is similar to construct $\mathrm{p} 150(1-508) / R P$ in Figure 1, except that it contains a stable stem-loop structure in the $5^{\prime}$ UTR of the Renilla luciferase cistron (see Zhou et al. 2001). (B) Yeast strain EGY48 $(\mathrm{CLONTECH})$ was transformed with this construct and grown in glucose-containing medium. Transformed cells were cultured for $24 \mathrm{~h}$ in glucose-containing medium, cells were washed with $\mathrm{H}_{2} \mathrm{O}$, and transcription of the dicistronic mRNA was induced by culturing in medium containing $2 \%$ galactose and $1 \%$ raffinose (indicated by arrow). Luciferase activities were determined at 24-h and 1-h intervals following galactose induction. The raw light units (rlu) are indicated as open circles $(\bigcirc)$ and a solid line for Renilla luciferase, and as solid squares (ם) and a hatched line for Photinus luciferase. To facilitate comparison, the Photinus and Renilla luciferase activities are plotted on different scales. The results indicated are from one experiment. Similar results were obtained when this experiment was repeated three times. Photinus luciferase activities did not increase in response to galactose when this experiment was performed using the control construct that lacks the TIF4631 5' leader. 
luciferase activities as low as $100 \mathrm{rlu} / \mu \mathrm{L}$ of lysate. In our studies, such numbers are indistinguishable from background; in our experiments, we obtained Renilla luciferase values that were up to several thousand-fold higher, ranging from $1 \times 10^{5}$ to $>1 \times 10^{6} \mathrm{rlu} / \mu \mathrm{L}$ of lysate. The low numbers reported by Verge et al. (2004) are cause for concern, because the first cistron should have been translated efficiently by a cap-dependent mechanism. In addition, the raw Photinus luciferase numbers are very similar; for example, construct 3 in CW04 and 334 cells (-/+ induction) yielded rlu's that were almost identical (1296/1226 and 1080/1067, respectively), suggesting that the luciferase activities may have reached a ceiling in these experiments.

In cell-free lysates, Verge et al. (2004) showed that the TIF4631 5' leader strongly repressed translation in a monocistronic context and did not function as an IRES. They did not, however, report testing their monocistronic constructs in cells. We did perform this experiment, and showed that the TIF4631 5' leader enhanced the production of a reporter enzyme (CAT) by sevenfold without altering the mRNA levels (Zhou et al. 2001). In other words, the translation efficiency was increased by sevenfold. Although this point needs no further discussion, it is important to note that our monocistronic mRNAs were transcribed from the powerful ADH promoter; if the TIF4631 5' leader contained a promoter capable of producing such reporter enzyme activities, it would have to be several times more active than the $\mathrm{ADH}$ promoter, making it one of the most active promoters ever described and easily detected. However, no transcripts from this promoter were detected in our studies.

The fact that the in vitro results obtained by Verge et al. (2004) differed from those obtained by us in living cells is not remarkable. Reasons for these differences may include problems with the integrity of the in vitro-transcribed templates or with their degradation in cell-free lysates. In addition, other factors can dramatically affect translation efficiencies in vitro, including the location of labile transacting factors within the cell, for example, nucleus versus cytoplasm, the integrity of trans-acting factors, and reaction conditions. In the literature, there are many examples where the results obtained in vitro do not mirror those obtained in living cells (e.g., Berthelot et al. 2004).

In summary, we conclude that Verge et al. (2004) have failed to convincingly demonstrate that the IRES activity observed with the eIF4G 5' leader is due to promoter activity. The putative promoter activity identified in their study does not colocalize with the IRES identified in our study. Moreover, the activity of this putative promoter is weak and cannot account for the reported IRES activities.

\section{REFERENCES}

Berthelot, K., Muldoon, M., Rajkowitsch, L., Hughes, J., and McCarthy, J.E. 2004. Dynamics and processivity of 40S ribosome scanning on mRNA in yeast. Mol. Microbiol. 51: 987-1001.

Goyer, C., Altmann, M., Lee, H.S., Blanc, A., Deshmukh, M., Woolford Jr., J.L., Trachsel, H., and Sonenberg, N. 1993. TIF4631 and TIF4632: Two yeast genes encoding the high-molecular-weight subunits of the cap-binding protein complex (eukaryotic initiation factor $4 \mathrm{~F}$ ) contain an RNA recognition motif-like sequence and carry out an essential function. Mol. Cell. Biol. 13: 4860-4874.

Verge, V., Vonlanthen, M., Masson, J.M., Trachsel, H., and Altmann, M. 2004. Localization of a promoter in the putative internal ribosome entry site of the Saccharomyces cerevisiae TIF4631 gene. RNA 10: $277-286$.

Zhou, W., Edelman, G.M., and Mauro, V.P. 2001. Transcript leader regions of two Saccharomyces cerevisiae mRNAs contain internal ribosome entry sites that function in living cells. Proc. Natl. Acad. Sci. 98: 1531-1536. 

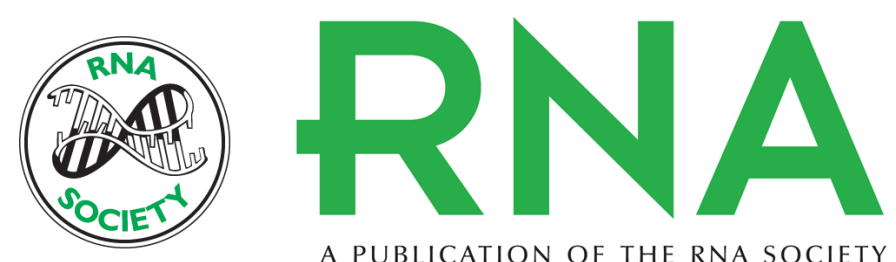

A PUBLICATION OF THE RNA SOCIETY

\title{
Reevaluation of the conclusion that IRES-activity reported within the $5^{\prime}$ leader of the TIF4631 gene is due to promoter activity
}

\author{
VINCENT P. MAURO, GERALD M. EDELMAN and WEI ZHOU
}

RNA 2004 10: 895-897

\section{Related Content \\ Answer to Reevaluation of the conclusion that IRES-activity reported within the $\mathbf{5 2}$ leader of the TIF4631 gene is due to promoter activity \\ MICHAEL ALTMANN, HANS TRACHSEL, MARTIN VONLANTHEN, et al. \\ RNA June , 2004 10: 898 \\ References This article cites 4 articles, 3 of which can be accessed free at: \\ http://rnajournal.cshlp.org/content/10/6/895.full.html\#ref-list-1 \\ Articles cited in: \\ http://rnajournal.cshlp.org/content/10/6/895.full.html\#related-urls \\ License \\ Email Alerting \\ Receive free email alerts when new articles cite this article - sign up in the box at the Service top right corner of the article or click here.}

To subscribe to $R N A$ go to:

http://rnajournal.cshlp.org/subscriptions 